\title{
LIPOMA DO CORPO CALOSO COM EXTENSÃO EXTRACRANIANA ATRAVÉS DE FALHA ÓSSEA FRONTAL
}

\author{
ROBERTO S. MARTINS*, ORILDO CIOUINI JR. * , \\ HAMILTON MATUSHITA**, JOSÉ PINDARO P. PLESE***
}

\begin{abstract}
RESUMO - Os lipomas intracranianos geralmente se localizam no corpo caloso e raramente apresentam expansão para a regiāo subgaleal. Revisão da literatura mostrou que apenas oito casos foram descritos em que o lipoma do corpo caloso se estendia para localização extracraniana atravês de falha óssea frontal. As manifestaçōes clínicas mais comuns nesses pacientes eram crises convulsivas e retardo mental. A literatura mostra que a ressecção do lipoma de corpo caloso geralmente leva a resultados catastróficos, devendo ser restrita a porçāo extracraniana do tumor. $O$ caso relatado é de uma criança portadora desta entidade, sem manifestação clínico-neurológica e que foi submetida a ressecção cirúrgica da porção subcutânea do lipoma com finalidade cosmética.
\end{abstract}

PALAVRAS-CHAVE: corpo caloso, lipoma intracraniano, osso frontal, falha óssea.

\section{Lipoma of the corpus callosum with extracranial extension through a frontal bone defect}

SUMMARY - Intracranial lipoma are usually localized in the corpus callosum and rarely extends to the subgaleal region. Only eight cases of lipoma of the corpus callosum with extracranial extension were reported in the literature. Seizures and mental retardation were the most common clinical findings in these patients. Data from the literature show that resection of intracranial lipoma has catastrophic results. The resection must be restricted to the extracranial portion. We report a child with lipoma of the corpus callosum with extracranial extension with no neurologic deficit submitted to resection of the extracranial extension.

KEY WORDS: corpus callosum, intracranial lipoma, frontal bone, bone defect.

O lipoma intracraniano é patologia pouco frequente sendo muitas vezes diagnosticado de forma incidental após necrópsia ${ }^{6}$. Topograficamente situam-se principalmente nas regiōes anteriores do corpo caloso e sua expansão para o espaço subgaleal através de orifício da tábua óssea é raro. Somente 8 casos desta patologia foram encontrados em revisão cuidadosa da literatura especializada, justificando portanto o relato deste caso.

\section{RELATO DO CASO}

AAS, paciente de 1 ano e 4 meses de idade, sexo masculino, nascido de parto a termo, com massa na região frontal esquerda (E), de crescimento lento e progressivo. O exame clínico neurológico revelava somente tumoração de consistência amolecida medindo $5 \times 5 \times 3 \mathrm{~cm}$ localizada na região frontal E, iniciando-se a partir da linha média, não pulsátil e que aumentava de volume com o choro.

Disciplina de Neurocirurgia, Departamento de Neurologia, Hospital das Clínicas, Faculdade de Medicina da Universidade de São Paulo (FMUSP): *Residente de Neurocirurgia; **Neurocirurgião; ***Professor Associado. Aceite: 11-abril-1995.

Dr. Roberto Sergio Martins - Divisão de Clínica Neurologica, Hospital das Clínicas, FMUSP - Av. Dr. Enéas de Carvalho Aguiar 255 - 05403-000 São Paulo SP - Brasil. 


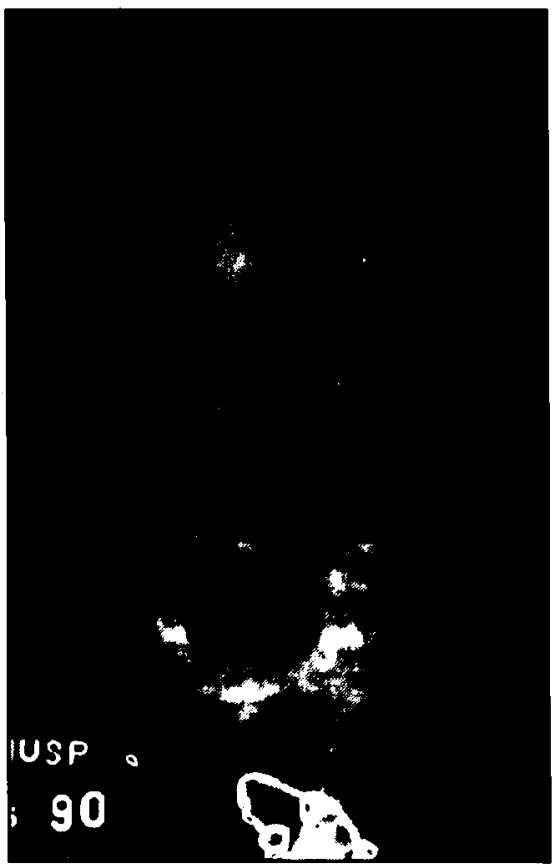

O estudo radiológico simples do crânio evidenciava falha óssea fronto-parietal $\mathrm{E}$, com $4 \mathrm{~cm}$ de diâmetro no seu maior eixo, iniciando-se a partir da linha média (Fig 1). $\mathrm{O}$ estudo encefálico por tomografia computadorizada de crânio (TC) mostrava lesão expansiva hipoatenuante e homogênea situada na região rostral do joelho do corpo caloso, com maior expansão à E (Fig 2). Esta tumoração se exteriorizava através de pertuito ósseo fronto-parietal $E$ acarretando abaulamento de partes moles da caixa craniana, sem hemiação encefálica (Fig 2). A infusão de contraste iodado propiciou realce da periferia da lesão. A angiografia cerebral bilateral mostrava zona hipoatenuante inter-hemisférica na região do joelho do corpo caloso que se prolongava até o lado $\mathrm{E}$ da fontanela bregmática, até o nível da falha óssea circular craniana. Evidenciava ainda estenose do seio sagital superior ao nivel da fontanela bregmática, elevação das artérias pericalosas na altura do joelho do corpo caloso e discreto desvio unilateral da artéria cerebral anterior direita (Fig 3). A artéria cerebral anterior $\mathrm{E}$ atravessava a zona hipoatenuante.

O paciente foi submetido a tratamento cirúrgico visando somente à ressecção da porção extradural da massa tumoral. O resultado do exame anátomo-patológico foi tecido gorduroso maduro compatível com lipoma.

Fig 1. Radiografia de crânio mostrando a falha óssea frontal.

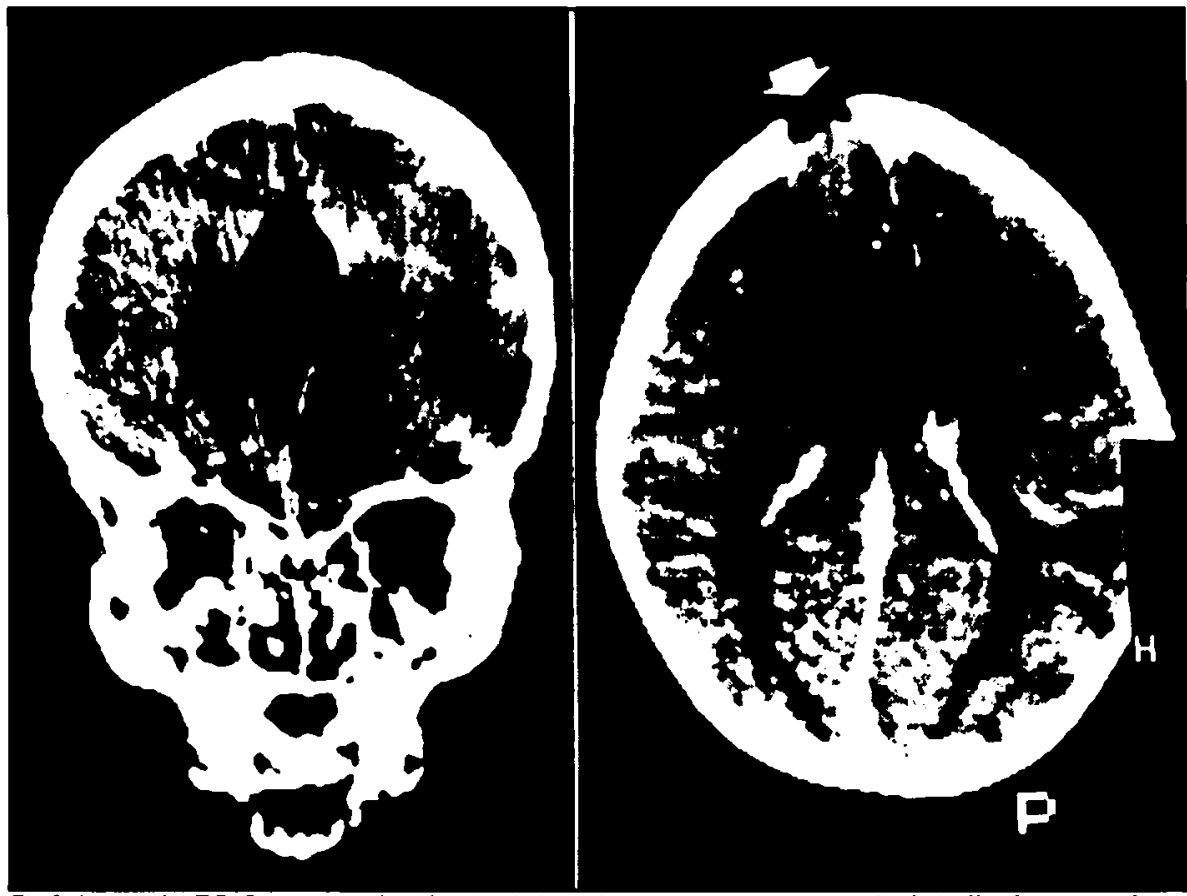

Fig 2. (esquerda) TC. Corte coronal evidenciando lesão hipoatenuante na topografia do joetho do corpo calosso. (direita) TC. Corte axial mostrando lesão hipoatenuante na topografia do joelho do corpo caloso, estendendose anteriormente até uma falha óssea frontal (seta). 


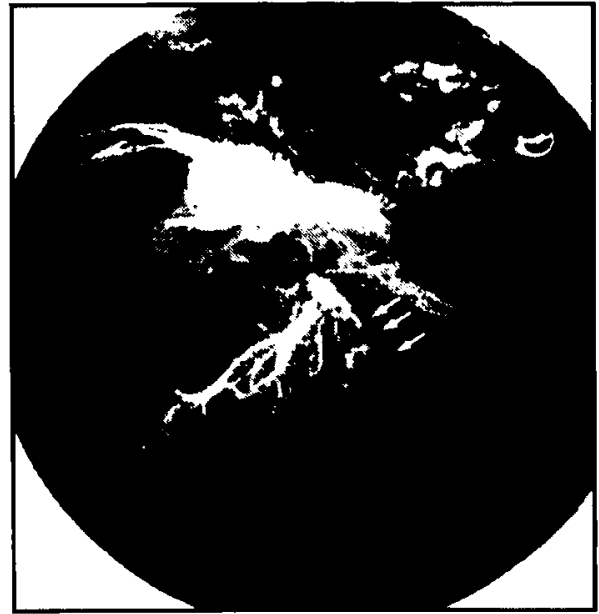

Fig 3. Angiografia cerebral (perfil) evidenciando deslocamento superior da artéria comunicante anterior e seus ramos (setas).
O paciente recebeu alta 5 dias após o ato cirúrgico e vem sendo acompanhado em ambulatório, com tempo de seguimento de 3 anos. As TC não mostram crescimento do lipoma intracraniano e a única queixa da mãe é excesso de agressividade da criança. $O$ exame neuropsicológico não mostra alterações significativas.

\section{COMENTÁRIOS}

A incidência do lipoma intracraniano é de menos de $0,1 \%$ dos tumores intracranianos. Kazner e col. analisaram os resultados do estudo tomográfico de 17500 pacientes num período de 4 anos nos hospitais universitáros de Munique, Mainz e Berlim, relatando incidência de $0,06 \%$ de pacientes com lipomas intracranianos 6 . Vonderahe e Niemer ${ }^{17}$ relataram 4 casos em 5000 autópsias, uma incidência de $0,08 \%$. Budka' após exame de 1956 necrópsias relatou 9 pacientes $(0,46 \%)$. Esta maior incidência foi justificada pela seleção dos casos, baseada na escolha de pacientes com patologias neurológicas.

Segundo List e col. ${ }^{10}$ e Kazner e col. ${ }^{6}$, no sistema nervoso central os lipomas são localizados mais frequentemente no corpo caloso, sendo também encontrados na região do tuber cinereum, lâmina quadrigêmia, cisterna ambiens, cisterna quiasmática, cisterna da fossa interpeduncular, cisterna silviana, cisterna pontina lateral, cisterna cerebelo-medular, tela coróidea do ventrículo lateral e do III ventrículo. Microscopicamente os lipomas são constituídos de células semelhantes à adipócitos adultos, sem evidências de transformação neoplásica ${ }^{12}$. Seu crescimento é lento e considerado como fazendo parte do crescimento dos tecidos como um todo. De acordo com Oftedal ${ }^{13}$ e Kushnet e Goldman ${ }^{7}$, em cerca de $50 \%$ dos casos o lipoma está associado com agenesia completa ou incompleta do corpo caloso e pode estar acompanhado de outras malformações relacionadas a estruturas da linha média como agenesia do vermis cerebelar, hipertelorismo, espinha bífida e crânio bífido ${ }^{10,21}$.

O quadro clínico do lipoma do corpo caloso é incaracterístico e cerca da metade dos casos são assintomáticos ${ }^{7,10}$. Quando presente, as manifestações clínicas mais comuns são crises convulsivas, cefaléia, hemiplegia, vômitos, labilidade emocional e retardo mental ${ }^{14}$.

Sosman e Campbell ${ }^{15}$, em 1946, foram os primeiros autores a caracterizar radiologicamente o lipoma do corpo caloso, sendo descrito na radiografia simples de crânio como zona de radiolucência aumentada, correspondendo às células gordurosas, na região do joelho do corpo caloso, circundada lateralmente por placas de calcificação lineares, geralmente bilaterais. Estas calcificaçōes apresentamse comumente como finas lâminas envol vendo o tumor, mas ocasionalmente podem atingir espessuras maiores $^{9} \mathrm{e}$, segundo Craddock e Riddervold ${ }^{3}$, podem ocorrer também no centro da lesão. Wolpert e col. ${ }^{19}$, em 1972, foram os primeiros autores a descrever os aspectos angiográficos desta patologia, caracterizados pelo desvio das artérias cerebrais anteriores, cujos ramos atravessam a lesão ou contornam a sua superfície. Segundo Wallace ${ }^{18}$, o lipoma do corpo caloso pode ainda se apresentar na angiografia somente como uma lesão avascular ou como imagem de fina trama de circulação patológica.

A partir da década de setenta o diagnóstico do lipoma intracraniano tornou-se mais frequente com o advento da TC. Neste exame, o lipoma do corpo caloso apresenta-se como uma área de baixa densidade, algumas vezes circundada por lâmina de material hiperatenuante, correspondendo às típicas calcificações ${ }^{6}$. De acordo com Kazner e col. ${ }^{6}$ e Zee e col. ${ }^{20}$, duas patologias, os cistos dermóides 
e os teratomas, podem apresentar características tomográficas semelhantes ao lipoma. O diagnóstico diferencial pode ser estabelecido pela localização preferencial, já que estas lesões tendem a ocorrer com maior frequência na região subfrontal e subtemporal e na região posterior do terceiro ventrículo. Além disso, estas massas tumorais geralmente apresentam-se como lesões heterogêneas, ao contrário dos lipomas, homogêneos na TC.

O tratamento do lipoma raramente é cirúrgico. Segundo Kazner e col. ${ }^{6}$, mesmo que operados, os pacientes não apresentam remissão das crises convulsivas, a manifestação clínica mais comum nesta patologia. Vários autores relataram taxa alta de morbidade e mortalidade após a remoção cirúrgica de lipomas de corpo caloso ${ }^{2}$, pois o tecido gorduroso constituinte do lipoma é ricamente vascularizado e confunde-se com o tecido fibroso que recobre as artérias pericalosas e seus ramos, dificultando a manipulação mesmo com o uso de técnicas microcirúrgicas ${ }^{6,21}$. Zettner e Netsky ${ }^{21}$, em 1960, relataram os resultados obtidos em 16 pacientes portadores de lipomas do corpo caloso após a resseç̧ão cirúrgica, o índice de mortalidade operatória sendo $62 \%$. Em todos os casos sobreviventes a ressecção cirúrgica foi parcial e $50 \%$ apresentaram piora neurológica em relação ao quadro inicial. Após este estudo outros autores relataram casos em que o óbito ocorreu após a resseç̧ão cirúrgica em pacientes portadores desta patologia ${ }^{14,16}$. Groff e col. ${ }^{4}, \mathrm{em} 1951$, relataram o único caso da literatura em que um lipoma do corpo caloso foi totalmente removido sem piora neurológica pós-operatória. $\mathrm{Na}$ opinião destes autores, esse tumor pode ser extirpado com sucesso se a ressecçāo não se expandir ao III ventrículo. Comentando os resultados desses autores, Kazner e col. ${ }^{6}$ argumentaram que, uma vez que o lipoma do corpo caloso na maioria dos casos está associado a agenesia total ou parcial do corpo caloso, é quase impossível não invadir o III ventrículo durante a sua ressecção cirúrgica. Estes mesmos autores, em 1980, relataram 11 casos de lipomas intracranianos, sendo em 3 casos de localização no corpo caloso. Em todos estes a abordagem foi cirúrgica, mas não foi especificado o grau de resseç̧ão ou a evolução pós-operatória.

A associaçāo de lipoma do corpo caloso e defeito ósseo frontal foi primeiramente descrita por Amold, citado por Zee e col. ${ }^{20}$, em 1868, seguido de mais 11 casos na literatura ${ }^{2,4-8,11-13,20}$. Destes, em 8 casos o lipoma se estendia através de uma falha óssea frontal até o espaço subcutâneo, onde formava uma lesão expansiva, com o diagnóstico histológico confirmado de lipoma ${ }^{2.5 .6 .8 .11 .12 .20}$. Zee $\mathrm{e}$ col. ${ }^{20}$, em 1981, relataram o único caso da literatura em que um lipoma do corpo caloso foi diagnosticado em associação com falha óssea frontal através da qual o lipoma se estendia até o espaço subcutâneo juntamente com tecido neural, constituindo uma verdadeira lipoencefalocele.

Nos 8 casos de lipoma do corpo caloso em continuidade com lipoma subcutâneo através de falha óssea frontal, o sintoma mais comum, além da protuberância frontal, foi crise convulsiva, presente em $60 \%$ dos pacientes, seguido de retardo mental em 2 casos (25\%). Hidrocefalia esteve presente associadamente em 2 casos (25\%). A manifestaçāo clínica do caso relatado neste estudo foi apenas a ocorrência de uma protuberância frontal, sendo também a forma de apresentação de um dos casos de Zee e col. ${ }^{20}$ e do caso descrito por Hayashi e col. ${ }^{5}$. O tratamento cirúrgico do lipoma de corpo caloso ocorreu nos casos relatados por Kinol e col. ${ }^{8}$, Nordin e col. ${ }^{12}$ e Kazner e col. ${ }^{6}$, com o óbito dos pacientes dos dois primeiros autores. Kazner e col. não informaram, em seu relato, o resultado cirúrgico e as condições pós-operatórias do paciente.

Nos casos de lipoma do corpo caloso em continuidade com lipoma subcutâneo através de falha óssea frontal, incluindo o relatado neste estudo, o curso clínico não parece ser diferente da evoluçāo dos pacientes com lipoma do corpo caloso sem outras lesões associadas. A literatura mostra que, devido ao prognóstico sombrio dos pacientes com lipoma do corpo caloso tratados cirurgicamente, a conduta nos casos em que esta lesão está em continuidade com lipoma frontal deve ser cirúrgica somente para a exérese da lesāo extracraniana com finalidade estética. 


\section{REFERÊNCIAS}

1. Budka H. Intracranial lipomatous hamartomas: a study of 13 cases including combinations with medulloblastomas, colloid and epidermoid cysts, angiomatosis and other malformations. Acta Neuropathol 1974, 28:205-222.

2. Cant WHP, Astley R. Lipoma of the corpus callosum. Arch Dis Childh 1952, 27:478-479.

3. Craddock WE, Riddervold HO. Unilateral calcification in a lipoma of the corpus callosus. J Can Assoc Radiol 1972, 23:192-194.

4. Groff RA, Liu CT, Leopold RL. Lipoma of the corpus callosum: survey of the literature and report of two surgical cases. Arch Neurol Psychiatr 1951, 65:253-254.

S. Hayashi T, Kadowaki T, Shyojima K, Honda E. Frontoethmoidal lipomeningocele. Child's Nerv Syst 1986, 2:37-39.

6. Kazner E, Stochdorph O, Wende S, Grumme T. Intracranial lipoma: diagnostic and therapeutic considerations. J Neurosurg 1980, 52:234-245.

7. Kushnet MW, Goldman RL. Lipoma of the corpus callosum associated with a frontal bone defect. Am J Roentgenol 1978, 131:517-518.

8. Kinol ME, Rasmussen G, Hamby WB. Lipoma of corpus callosum. J Neuropathol Clin Neurol 1951, 1:168-178.

9. Larsen JL, Stiris G. Lipoma of the corpus callosum with atypical calcification. Br J Radiol 1970, 43:576-577.

10. List CF, Holt JF, Everett M. Lipoma of the corpo callosum: a clinicopathologic study. Am J Roentgenol Radium Ther Nucl Med 1946, 55:125-134.

11. Luten PJ. Lipomen van het corpus callosum. Ned Tijdschr Geneeskd 1951, 95:1416-1421.

12. Nordin WA, Tesluk H, Jones RK. Lipoma of the corpus callosum. Arch Neurol Psychiatr 1955, 74:300-307.

13. Oftedal S. Anomalies of the mid-line structures of the brain. Acta Psychiatr Neurol Scand 1959, 34:451-453.

14. Patel AN. Lipoma of the corpus callosum: a nonsurgical entity. NC Med J 1965, 26:328-335.

15. Sosman MC, Campbell JA. Mid-line anomalies of the brain: their diagnosis by pneumoencephalography. Radiology 1946, 46:119-131.

16. Sukthomya C, Menakanit V. Lipoma of the nervous system: report of 2 cases. Australasian Radiology $1973,17: 256-260$.

17. Vonderahe AR, Niemer WT. Intracranial lipoma: a report of four cases. J Neuropathol Exp Neurol 1944, 3:344-354.

18. Wallace D. Lipoma of the corpus callosum J Neurol Neurosurg Psychiatry 1976, 39:1179-1185.

19. Wolpert SM, Carter BL, Ferris EJ. Lipoma of the corpus callosum: an angiographic analysis. Am J Roentgenol 1972, 115:92-99.

20. Zee C, McComb JG, Segall HD, Tsai FY, Stanley P. Lipomas of the corpus callosum associated with frontal dysraphism. J Comput Assist Tomogr 1981, 5:201-205.

21. Zettner A, Netsky MG. Lipoma of the corpus callosum. J Neuropathol Exp Neurol 1960, 19:305-319. 